\title{
Retrospective analyzes of adverse events during biologic agents in children with juvenile idiopathic arthritis from a single center in Turkey
}

\author{
Sibel Balci ${ }^{1}$, ilksen Demir ${ }^{2}$, Mahir Serbes ${ }^{3}$, Dilek Doğruel ${ }^{3}$, Derya Ufuk Altıntaş3, \\ Rabia Miray Kışla Ekinci ${ }^{1}$ \\ 1Department of Pediatric Rheumatology, Cukurova University Faculty of Medicine, Adana, Turkey \\ ${ }^{2}$ Department of Pediatrics, Cukurova University Faculty of Medicine, Adana, Turkey \\ ${ }^{3}$ Department of Pediatric Allergy and Immunology, Cukurova University Faculty of Medicine, Adana, Turkey
}

\begin{abstract}
Objectives: Juvenile idiopathic arthritis is the most common rheumatic disease in childhood. Biologic agents have changed the course of juvenile idiopathic arthritis. However, there are concerns regarding the occurrence of serious adverse events in patients receiving biologic agents. The aim of this study was to evaluate adverse events in children with juvenile idiopathic arthritis receiving biologic agents.

Material and methods: This retrospective study includes juvenile idiopathic arthritis patients receiving biologic agents. Demographic features and adverse events during biologic agents were collected from medical files. Adverse events that either resulted in death, were life-threatening, required inpatient hospitalization, or resulted in persistent or significant disability/incapacity were considered as serious adverse events.

Results: In total, 162 juvenile idiopathic arthritis patients (55.6\% female) receiving biologic agents were enrolled: 101 (62.3\%) patients treated with etanercept, 27 (16.7) with tocilizumab, 14 (8.6\%) with adalimumab, 15 (9.2\%) with anti-interleukin 1 agents (13 canakinumab, 2 anakinra), and 5 (3.1\%) with infliximab. $75.9 \%$ of the patients received concomitantly disease-modifying anti-rheumatic drugs, and $20.4 \%$ received disease-modifying anti-rheumatic drugs plus corticosteroid. The mean age at initiation of the biologic agent was $10.5 \pm 4.3$ years. The mean age at the study enrolment was $12.1 \pm 4.5$ years. The mean follow-up duration was $19.7 \pm 2.1$ months. The most frequent adverse event was upper respiratory tract infections (54.3\%) followed by urinary tract infections (21\%). Anaphylaxis occurred in 3 patients (1.9\%): 2 with tocilizumab and one with infliximab. Macrophage activation syndrome occurred in 1 patient $(0.6 \%)$ receiving tocilizumab. Lung tuberculosis developed in 2 patients $(1.2 \%)$ receiving canakinumab. The frequency of serious adverse events in total was $6.7 \%$.

Conclusions: While the most frequent adverse events during biologic agents was upper respiratory tract infections, the frequency of serious adverse events was $6.7 \%$; therefore, juvenile idiopathic arthritis patients receiving biologic agents should be carefully evaluated for these adverse events in clinical practice.
\end{abstract}

Key words: juvenile idiopathic arthritis, biologic agents, infections, adverse events.

\section{Introduction}

Juvenile idiopathic arthritis (JIA) is the most common rheumatic disease in childhood with a chronic course. Over the past decades, pharmacotherapy of JIA has improved dramatically with the utilization of biologic agents (BAs) that control the disease activity much more effectively, thus improving the quality of life of the patients [1-3].

Biologic agents are a new sort of drugs that differ from non-biologic disease-modifying antirheumatic drugs (DMARDs) in that they are manufactured with 
biologic processes and target specific molecules that are expressed on cells or secreted into the extracellular space, such as tumour necrosis factor $\alpha$ (TNF- $\alpha$ ), interleukin (IL) $1 \beta$, and IL-6 [4].

Essentially, all biologics are immunogenic because they are non-self; therefore, they may lead to antibody responses that give rise to neutralization of biologic activity, anaphylactoid reactions, and loss of efficacy. These effects seem to be related with host-related factors, the dose, and the route of administration [5].

There are several studies investigating the efficacy and safety of BAs with various results [6-11]. The most frequent adverse event on BAs is upper respiratory tract infections (URTIS) [6-11].

There are also concerns about the association between occurrence of serious infections, malignancies, and autoimmune diseases with exposure to BAs [4]. However, due to the difficulty of a randomized placebocontrolled study design, clinical trials are disempowered to determine whether serious infections, malignancies, and autoimmune diseases are associated with BAs [4].

Therefore, the data on the long-term safety of BAs in children are still scarce. The objective of the current study is to retrospectively analyze the adverse events in JIA patients on BAs from a pediatric rheumatology center.

\section{Material and methods}

This is a retrospective study investigating adverse events during BA treatment in children with $\|$ A. Juvenile idiopathic arthritis patients diagnosed between August 2008 and March 2019, who were still in follow-up in our pediatric rheumatology department, were included in the study.

Diagnosis of the patients and treatment choices were designated by pediatric rheumatologists accordingly. The patients were diagnosed according to the International League of Associations for Rheumatology (ILAR) classification criteria and sub-grouped as oligoarticular JIA, rheumatoid factor (RF)-positive polyarticular JIA, RF-negative polyarticular JIA, systemic-onset JIA (soJIA), and enthesitis-related arthritis (ERA) [12].

All patients' data were collected from the medical files of the patients. Demographic characteristics, treatment modalities, laboratory data of the patients, and the diagnosis of rheumatic disease in first-degree relatives were included in the data. Prior to study initiation, written informed consent was obtained from all the patients' parents. Ethical approval was obtained from the Institutional Review Board of the local medical school.

The biologic agents that were received by the participants consisted of TNF- $\alpha$ inhibitors (etanercept, adalimumab, and infliximab), fully human monoclonal antibody targeting IL-1 $\beta$ (canakinumab), IL-1 receptor antagonist (anakinra), and humanized monoclonal antibody targeting IL-6 receptor (tocilizumab). Pediatric rheumatologists selected the BAs in agreement with type and activity of the disease and switched or discontinued the BAs due to adverse events or remission. The patients receiving BAs were followed up regularly by pediatric rheumatologists according to JIA subgroup, disease activity, and the type of BAs, in at least threemonth intervals.

Adverse events (AEs) and serious adverse events (SAEs) were recorded from the medical files of the patients, which included comprehensive information about adverse events that were based on interrogation of symptoms, physical examination, and laboratory parameters at least quarterly during routine follow-up. Adverse events that either resulted in death, were life-threatening, required inpatient hospitalization, or resulted in persistent or significant disability/incapacity were considered as SAEs [13].

Upper respiratory tract infections (URTIS) included rhinosinusitis, pharyngitis, laryngitis, laryngotracheitis, and otitis media, which were treated in outpatient clinics. Pneumonia referred to lower respiratory tract infection with symptoms included fever, chills, and cough with sputum production and confirmed by $X$-ray testing. Anaphylaxis was defined according to National Institutes of Health Food Allergy and Anaphylaxis Network criteria for anaphylaxis [14].

Local injection site reactions (ISRs) were defined as having redness, itching, pain, swelling, and burning at the injection site. However, injection site reactions more than $4 \mathrm{~cm}$ in diameter were defined as large local reactions, which were also considered as SAEs. Microscopic analyses of midstream urine samples were used to determine urinary tract infections (UTIS). The finding of $>5$ white blood cells per high-power field in a centrifuged urine specimen in a symptomatic patient was reported as a UTI.

\section{Statistical analysis}

All statistical analyses were performed by using Statistical Package for the Social Sciences (SPSS) software version 20.0 (IBM SPSS Statistics). Demographic features and disease characteristics were summarized with the use of descriptive statistics.

Categorical variables were presented as numbers and percentages. Continuous variables were summarized as mean and standard deviation and as median and minimum-maximum where appropriate. $\chi^{2}$ test was performed to compare frequencies of AEs among the administered BAs. The statistical level of significance for all tests was determined to be 0.05 . 


\section{Results}

\section{Baseline characteristics}

A total of 162 juvenile idiopathic arthritis patients were included in the present study, of whom 90 (55.6\%) were female and 72 (44.4\%) were male. While the mean age at the initiation of a biologic agent was $10.5 \pm 4.3$ years, the mean age at the study enrolment was $12.1 \pm 4.5$ years. The mean follow-up duration was $19.7 \pm 2.1$ months. The total duration of exposure to BAs was 269.7 years. Demographic features of the disease are given in Table I.

The distribution of juvenile idiopathic arthritis patients in agreement with International League of Associations for Rheumatology (ILAR) criteria were as follows: oligoarticular JA ( $n=53,32.7 \%)$, systemic onset JIA (soJA, $n=51,31.5 \%)$, RF-negative polyarticular JIA ( $n=28$, $17.3 \%)$, ERA ( $n=24,14.8 \%)$, and RF-positive polyarticular JIA $(n=6,3.7 \%)$. The distribution of the disease subgroups is given in detail in Table I.

\section{Biological agents and concomitantly administered immunosuppressive drugs}

Etanercept was the most administered BA, with a frequency of $62.3 \%(n=101)$. Moreover, TNF- $\alpha$ antagonists represented $70.9 \%(n=115)$ of all BAs. The median administered dosage of all BAs was 31 (range; 1-520) doses. A total of 123 (75.9\%) patients were given DMARDs concomitantly, particularly methotrexate. Moreover, $20.4 \%(n=33)$ of the patients were prescribed both corticosteroid and DMARDs.

The distribution of BAs and concomitantly administered immunosuppressive drugs are given in Table I.

\section{Adverse events}

The most frequently encountered adverse events were upper respiratory tract infections, with a 54.3\% $(n=88)$ frequency, which were treated in an outpatient clinic. Ten patients (6.2\%) developed pneumonia, which required hospitalization in 3 patients. While urinary tract infections occurred in 34 (21\%) patients, none of them developed pyelonephritis.

Twenty-four (17.8\%) out of 135 patients developed injection-site reactions with BAs administered subcutaneously. Injection-site reactions were below $4 \mathrm{~cm}$ in diameter and were treated in an outpatient clinic, except for the two patients having etanercept.

Etanercept treatments were discontinued due to the large ISRs in those two patients. Throughout the followup, anaphylactic reactions occurred in two patients having tocilizumab and in one patient having infliximab (a total of 3 patients, 1.9\%), which led to discontinuation
Table I. Demographic features and distribution of the disease subgroups and administered biologic agents of juvenile idiopathic arthritis patients

\begin{tabular}{|c|c|}
\hline Parameters & Numerical values \\
\hline Demographic features & $162(100)$ \\
\hline Female/male, $n(\%)$ & $90(55.6) / 72(44.4)$ \\
\hline $\begin{array}{l}\text { The mean age of biologic treatment } \\
\text { onset, years (SD) }\end{array}$ & $10.5(4.3)$ \\
\hline The mean age at study time, years (SD) & $12.1(4.5)$ \\
\hline Mean follow-up duration, months (SD) & $19.7(2.1)$ \\
\hline $\begin{array}{l}\text { Median administered dose of biologic } \\
\text { agents; minimum-maximum }\end{array}$ & $31(1-520)$ \\
\hline \multicolumn{2}{|l|}{ JIA subgroups } \\
\hline Oligoarticular JIA, $n(\%)$ & $53(32.7)$ \\
\hline Systemic-onset JIA, $n(\%)$ & $51(31.5)$ \\
\hline RF-negative polyarticular JIA, $n(\%)$ & $28(17.3)$ \\
\hline Enthesitis-related arthritis JIA, $n(\%)$ & $24(14.8)$ \\
\hline RF-positive polyarticular JIA, $n(\%)$ & $6(3.7)$ \\
\hline \multicolumn{2}{|l|}{ Biologic agents } \\
\hline Etanercept, $n(\%)$ & $101(62.3)$ \\
\hline Tocilizumab, n (\%) & $27(16.7)$ \\
\hline Adalimumab, $n$ (\%) & $14(8.6)$ \\
\hline Canakinumab, $n(\%)$ & $13(8)$ \\
\hline Infliximab, $n(\%)$ & $5(3.1)$ \\
\hline Anakinra, $n(\%)$ & $2(1.2)$ \\
\hline \multicolumn{2}{|l|}{ Co-prescriptions with biologic agents } \\
\hline DMARDs, $n(\%)$ & $123(75.9)$ \\
\hline DMARDs plus corticosteroid, $n(\%)$ & $33(20.4)$ \\
\hline
\end{tabular}

DMARDs - disease-modifying antirheumatic drugs, JIA - juvenile idiopathic arthritis, RF-rheumatoid factor, SD - standard deviation.

of those BAs. An episode of macrophage activation syndrome (MAS) occurred in one JIA patient on tocilizumab treatment.

Moreover, two siblings with solIA having canakinumab were diagnosed with lung tuberculosis. Serious adverse events were as follows: 1 MAS episode in a solIA patient, 3 anaphylactic reactions in 1 oligoarticular JIA and in 2 soJIA patients, 2 lung tuberculosis in sollA patients, and 3 pneumonia cases that required hospitalization in SolIA patients, and 2 large local injection site reactions occurred in two ERA patients on BAs.

In total, serious adverse events occurred with a frequency of $6.7 \%(n=11)$ among JIA patients on BAs. None of the patients developed malignancy or died during BA treatment due to AEs. The frequency of all adverse events during BAs in JIA patients are given in Table II, in detail. 
Table II. The frequency of adverse events during biological drug therapy in children with juvenile idiopathic arthritis

\begin{tabular}{|lc|}
\hline Parameters & Numerical values \\
\hline Infections & $88(54.3)$ \\
\hline Upper respiratory tract infections, $n$ (\%) & $34(21)$ \\
\hline Urinary tract infections, $n(\%)$ & $17(10.5)$ \\
\hline Herpes labialis, $n$ (\%) & $10(6.2)$ \\
\hline Pneumoniae, $n(\%)$ & $2(1.2)$ \\
\hline Tuberculosis, $n(\%)$ & $2(1.2)$ \\
\hline Abscess, $n(\%)$ & $1(0.6)$ \\
\hline Impetigo, $n(\%)$ & $1(0.6)$ \\
\hline Chickenpox, $n(\%)$ & $12(7.4)$ \\
\hline Hematologic events & $6(3.7)$ \\
\hline Lymphopenia, $n(\%)$ & $2(1.2)$ \\
\hline Neutropenia, $n(\%)$ & $1(0.6)$ \\
\hline Thrombocytopenia, $n(\%)$ & $24(17.8)$ \\
\hline Haemolytic anaemia, $n(\%)$ & $5(3.1)$ \\
\hline Others & $5(3.1)$ \\
\hline Injection site reactions, $n(\%)$ & $3(1.9)$ \\
\hline Erythematous skin rashes, $n(\%)$ & $1(0.6)$ \\
\hline Elevated liver function tests, $n(\%)$ & \\
\hline Anaphylaxis, $n(\%)$ & \\
\hline Macrophage activation syndrome, $n(\%)$ & $(\%)$ \\
\hline
\end{tabular}

Table III. Tuberculin skin test results and the frequency of isoniazid preventive therapy of juvenile idiopathic arthritis patients on biologic agents

\begin{tabular}{|lc|}
\hline Parameters & Numerical values \\
\hline Initial TST, median (min-max) & $0(0-20)$ \\
\hline Last TST, median (min-max) & $0(0-20)$ \\
\hline IPT, $n(\%)$ & $23(14.2)$ \\
\hline Tuberculosis, $n(\%)$ & $2(1.2)$ \\
\hline
\end{tabular}

TST - tuberculin skin test, IPT - isoniazid preventive therapy.

The median initial tuberculin skin test result was 0 (range; 0-20). After administration of BAs, isoniazid preventive therapy (IPT) was given to 23 (14.2\%) JIA patients due to increased tuberculin skin test (TST).

Moreover, two siblings with sollA developed lung tuberculosis during canakinumab treatment and were treated accordingly, which resulted in restarting BAs. Those two solIA patients were treated with etanercept, abatacept, and tocilizumab before canakinumab treatment.
Tuberculin skin test results and the frequency of isoniazid preventive therapy of JIA patients on BAs are given in Table III.

For comparison of the frequency of adverse events, the patients were grouped according to the administered BAs as follows:

- group 1: anti-TNF- $\alpha$ agents (etanercept, adalimumab and infliximab),

- group 2: anti-IL-1 agents (canakinumab and anakinra), - group 3: tocilizumab.

The frequency of adverse events did not differ between the groups except for lung tuberculosis and neutropenia. The frequency of neutropenia was significantly higher in JIA patients on tocilizumab and the frequency of lung tuberculosis in patients on canakinumab.

Moreover, the frequency of co-treatment with corticosteroid and disease-modifying anti-rheumatic drugs was significantly higher in the groups using anti-IL-1 agents and tocilizumab. Comparison of the frequency of adverse events between juvenile idiopathic arthritis patients according to the administered BAs are given in Table IV.

In the present study, we could not compare the frequency of AEs in patients according to the JIA subgroups due to the small number of some subgroups.

\section{Discussion}

In the present study, adverse events during BAs were retrospectively investigated in JIA patients. The most utilized biologic agents were anti-TNF- $\alpha$ agents, and the most encountered adverse event was URTIS in JIA patients on BAs in this study. The second most encountered adverse event was UTIs in which none of them developed pyelonephritis and required hospitalization.

In total, serious adverse events occurred with a frequency of $6.7 \%(n=11)$ among JIA patients on BAs - in detail: 1 MAS episode in a sollA patient on tocilizumab, 3 anaphylactic reactions in 1 oligoarticular JIA on infliximab and in 2 soJIA patients on tocilizumab, 2 lung tuberculosis in sollA patients on canakinumab, and 3 pneumonia that required hospitalization in sollA patients on tocilizumab, and 2 large local injection site reactions in 2 ERA patients on etanercept occurred. Although none of the patients developed malignancy or died during BAs treatment due to AEs, the short length of the study period makes the interruption difficult in this regard.

Up to now, there have been two observational retrospective studies investigated all BAs, 1 in JIA patients and the other in all pediatric rheumatic diseases [11, 15].

Cabrera et al. [11] reported a multicenter, observational, retrospective study investigating the incidence of side effects of biological agents in 813 pediatric patients with inflammatory diseases. The main diagnosis 
Table IV. Comparison of the frequency of adverse events between juvenile idiopathic arthritis patients according to the administered biologic agents

\begin{tabular}{|c|c|c|c|c|}
\hline Parameter & $\begin{array}{c}\text { Anti-TNF- } \alpha \text { agents } \\
120(74.1)\end{array}$ & $\begin{array}{c}\text { Anti-IL-1 agents } \\
15(9.3)\end{array}$ & $\begin{array}{c}\text { Tocilizumab } \\
27(16.7)\end{array}$ & $p$-value \\
\hline \multicolumn{5}{|l|}{ Co-prescriptions } \\
\hline Co-prescription of DMARDs, $n(\%)$ & $87(72.5)$ & $11(73.3)$ & $25(92.5)$ & 0.066 \\
\hline Co-prescription of DMARDs + CS, $n(\%)$ & $13(10.8)$ & $6(40.4)$ & $14(51.8)$ & 0.001 \\
\hline \multicolumn{5}{|l|}{ Adverse events } \\
\hline URTI, $n(\%)$ & $67(55.8)$ & $6(40)$ & $15(55.6)$ & 0.505 \\
\hline UTIs, $n(\%)$ & $21(17.5)$ & $6(40)$ & $7(25.9)$ & 0.103 \\
\hline Herpes labialis, $n(\%)$ & $13(10.8)$ & $1(6.7)$ & $3(11.1)$ & 0.878 \\
\hline Pneumoniae, $n$ (\%) & $5(4.2)$ & $2(13.3)$ & $3(11.1)$ & 0.192 \\
\hline Tuberculosis, $n(\%)$ & $0(0)$ & $2(13.3)$ & $0(0)$ & 0.001 \\
\hline Abscess, $n(\%)$ & $1(0.8)$ & $0(0)$ & $1(3.7)$ & 0.428 \\
\hline Impetigo, $n(\%)$ & $1(0.8)$ & $0(0)$ & $0(0)$ & 0.839 \\
\hline Chickenpox, $n(\%)$ & $0(0)$ & $0(0)$ & $1(3.7)$ & 0.081 \\
\hline ISRs, $n(\%)$ & $20(16.7)$ & $3(20)$ & $0(0)$ & 0.193 \\
\hline Erythematous skin rashes, $n(\%)$ & $3(2.5)$ & $0(0)$ & $2(7.4)$ & 0.316 \\
\hline Elevated LFTs, $n(\%)$ & $4(3.3)$ & $0(0)$ & $1(3.7)$ & 0.765 \\
\hline Anaphylaxis, $n$ (\%) & $1(0.8)$ & $0(0)$ & $2(7.4)$ & 0.062 \\
\hline MAS, $n(\%)$ & $0(0)$ & $0(0)$ & $1(3.7)$ & 0.081 \\
\hline Neutropaenia, $n$ (\%) & $0(0)$ & $1(6.7)$ & $5(18.5)$ & 0.001 \\
\hline Lymphopaenia, $n(\%)$ & $8(6.7)$ & $2(13.3)$ & $2(7.4)$ & 0.649 \\
\hline Thrombocytopaenia, $n$ (\%) & $1(0.8)$ & $0(0)$ & $1(3.7)$ & 0.428 \\
\hline
\end{tabular}

CS - corticosteroid, DMARDs - disease-modifying antirheumatic drugs, ISRs - injection-site reactions, LFTs - liver function tests,

MAS - macrophage activation syndrome, URTI - upper respiratory tract infection, UTIs - urinary tract infections. $\chi^{2}$ test was utilised to compare the frequency of adverse events among the three groups, significant $p$-values $(<0.05)$ are presented in bold.

in that study was JIA with $84 \%$ frequency. The mean follow-up duration was $4.7 \pm 3.1$ years. There was a total of 1179 BA prescriptions for 813 patients. The anti-TNF- $\alpha$ agents, particularly etanercept, represented $75 \%$ of all utilized BAs. A total of 419 adverse events reported in 222 patients. Adverse events were most frequently mild (46\%). The frequency of severe and very severe adverse events was $15 \%$ in all patients. The incidence of adverse events was higher in JIA patients. There were two MAS episodes during tocilizumab treatment. Moreover, two serious events during etanercept treatment - one Hodgkin's lymphoma in a RF-positive JIA patient and one death due to JIA associated pulmonary fibrosis - occurred. Furthermore, one severe sepsis requiring intensive care unit hospitalization in the non-JIA group occurred during infliximab treatment, and one demyelinating lesion appeared with canakinumab. It was concluded that the study found an overall favorable outcome for children with pediatric inflammatory rheumatic diseases treated with all BAs [11].
The other study was from Finland by Tarkiainen et al. [15]. The study included 348 consecutive patients with JIA from three centers. Oligoarticular juvenile idiopathic arthritis was the most frequent disease subgroup, and etanercept was the most utilized biologic agent. Upper respiratory tract infections were the most encountered adverse event (71.8\%). A total of 121 patients (35\%) experienced SAEs and $12.6 \%$ of them were serious infections. Moreover, two systemic-onset juvenile idiopathic arthritis patients on etanercept died due to serious infections. None of the patients developed lung tuberculosis and malignancy [15]. Similarly to both studies, in our study, URTIs were the most encountered adverse events during BAs. The rate of serious adverse events in the current study was $6.7 \%$, with no sequelae or death after discontinuation of BAs, similar to the report of Cabrera et al. [11]; however, the result was lower than the Finnish study in which SAEs were reported in 35\% of the patients [15].

The difference between the rates of SAEs among studies might be due to the methodology of the studies. 
In the Finnish study [15], at least three sources of information were used for collecting data, which may have led to more accurate data on AEs as compared to our study, in which data collection were based on medical records held by pediatric rheumatologists, similar to the report of Cabrera et al. [11]. Moreover, another explanation for the differences might be the diversity of definition of SAEs. In the Finnish study [15], adverse events, classified as serious according to the Common Terminology Criteria for Adverse Events (CTCAE) [16], which contain various organ infections and symptoms, psychiatric disorders, even elevated liver transaminase levels, neutropenia which may also lead to increased frequency of SAEs compare to our study, in which AEs either resulted in death, were life-threatening, required inpatient hospitalization, resulted in persistent or significant disability/ incapacity were considered as SAEs.

Serious adverse events in the present study included anaphylaxis in 3 patients, large ISRs in 2 patients, tuberculosis in 2 patients, MAS in 1 patient, and pneumonia that required hospitalization in 3 patients.

While biologic agents have revolutionized the therapies for chronic inflammatory, neoplastic, and autoinflammatory disease, like other pharmaceutical agents, they can cause anaphylactic reactions. Anaphylaxis has been reported with various BAs, including rituximab, infliximab, and tocilizumab in pediatric rheumatic diseases [17-21]. Anaphylactic reactions to BAs can occur on the first dose or after multiple exposures [22].

In the present study we encountered anaphylaxis in 3 patients -2 during tocilizumab and 1 during infliximab treatment. Anaphylaxis was reported in 4 (1.1\%) patients during infliximab treatment in the study of Tarkiainen et al. [15]. To our knowledge, there is only one study evaluating severe hypersensitivity reactions to biological agents in children with all rheumatic diseases from Turkey [23].

In that study, 128 patients using eight different BAs with all rheumatic diseases were evaluated. The frequency of anaphylaxis was reported to be $3.9 \%$ in children with all rheumatic diseases and $4 \%$ in JIA patients [23]. In the present study, the frequency of anaphylaxis was lower (1.9\%) than the previous report among JIA patients. Unlike our study, the previous study enrolled patients with all rheumatic diseases, which might explain the higher frequency of anaphylaxis among patients receiving BAs in the previous study.

The most important complication of the solIA is MAS, and it can be deadly. Macrophage activation syndrome occurs in almost $10 \%$ of solIA patients and may occur even under BAs [24-26]. In the present study we encountered one MAS episode at the sixth dose of tocilizumab treatment in a soJIA patient. In regard to mac- rophage activation syndrome, Cabrera et al. [11] also reported two MAS episodes during tocilizumab treatment in JIA patients. However, in the other previous study, they did not report any MAS episode during follow-up, rather than SoJIA activation [15].

The risk of tuberculosis development or activation is an area of concern in BA-administered patients [27]. In regard to tuberculosis, in this study 2 siblings with soJIA under canakinumab treatment were diagnosed with lung tuberculosis. However, they were also given methotrexate, methylprednisolone concomitantly, and had a long-lasting disease that led to being given various BAs before canakinumab treatment. Therefore, it is difficult to conclude that these side effects are solely related to canakinumab administration.

There are diverse clinical studies investigating the safety of BAs as well as the effectiveness of BAs in the treatment of pediatric rheumatic diseases, particularly JIA [6-11]. In those studies, BAs were found to be well tolerated and the most encountered infections were the URTIs which were treated at outpatient clinics. In the present study, the most frequent AEs were also URTIS with a frequency of $54.3 \%$, which were also treated at outpatient clinics, similarly to the literature.

However, there is only one prospective study investigating the occurrence of infections in JIA patients on all BAs from Turkey [6]. A total of 307 juvenile idiopathic arthritis patients, mainly oligoarticular JIA, were included the study. Juvenile idiopathic arthritis patients on BAs were examined by a pediatric infectious disease specialist every 2 months during 1 year. The most frequently administered BA was etanercept. During the study period, $57 \%$ of the patients developed infection. Upper respiratory tract infections were the most frequently encountered infections, in which none of the patients required hospitalization. The rate of urinary tract infections was similar to our findings, with $18.8 \%$ frequency. Chickenpox was diagnosed in 7 patients (2.2\%), none of whom were hospitalized or developed any complications. During one-year follow-up, pneumoniae was diagnosed in 12 patients (3.9\%), in whom two required hospitalization. Lung tuberculosis occurred in 2 patients $(0.6 \%)$ during etanercept and adalimumab treatments. The findings of our study and the previous study are comparable in terms of encountered infections, and most of the encountered AEs were easily treated at outpatient clinics.

The main limitation of the present study is the retrospective design, which might lead to miss-collection of some adverse events. Moreover, the relatively small number of each BA group and juvenile idiopathic arthritis subgroups make the interpretation of statistical analyses difficult between groups. Therefore, further, multicenter, prospective studies are needed for the iden- 
tification of the real-life incidence of side effects of BAs in pediatric rheumatic diseases. However, we still think that the data on SAEs give us important knowledge about JIA patients on BAs.

\section{Conclusions}

In the current study, upper respiratory tract infections were the most frequently encountered adverse event in IIA patients using BAs. In total, the frequency of SAEs was $1.9 \%$. Although serious adverse events are rare in clinical practice and can be managed easily either with discontinuation of therapy or with treatment of AEs, we suggest that patients on BAs should be monitored and interrogated for the possibility of various adverse events.

\section{Acknowledgements}

The authors would like to thank Prof. Dr. Mustafa Yılmaz for valuable input in this study.

The authors declare no conflict of interest.

\section{References}

1. Poddighe D, Romano M, Gattinara M, Gerloni V. Biologics for the treatment of juvenile idiopathic arthritis. Curr Med Chem 2018; 25: 5860-5893, DOI: 10.2174/0929867325666180522085716.

2. Horneff G. Safety of biologic therapies for the treatment of juvenile idiopathic arthritis. Expert Opin Drug Saf 2015; 14: 1111-1126, DOI: 10.1517/14740338.2015.1042453.

3. Guzman J, Oen K, Tucker LB, et al. The outcomes of juvenile idiopathic arthritis in children managed with contemporary treatments: results from the ReACCh-Out cohort. Ann Rheum Dis 2015; 74: 1854-1860, DOI: 10.1136/annrheumdis-2014-205372.

4. Ilowite NT, Laxer RM. Pharmacology: biologics. In: Textbook of Pediatric Rheumatology. Petty RE, Laxer RM, Lindsley CB, Wedderburn LR (eds.). Elsevier, Philadelphia 2016: 161-175.

5. Strand V, Kimberly R, Isaacs JD. Biologic therapies in rheumatology: lessons learned, future directions. Nat Rev Drug Discov 2007; 6: 75-92, DOI: 10.1038/nrd2196.

6. Aygun D, Sahin S, Adrovic A, et al. The frequency of infections in patients with juvenile idiopathic arthritis on biologic agents: 1-year prospective study. Clin Rheumatol 2019; 38 1025-1030, DOI: 10.1007/s10067-018-4367-9.

7. Windschall D, Müller T, Becker I, Horneff G. Safety and efficacy of etanercept in children with the JIA categories extended oligoarthritis, enthesitis-related arthritis and psoriasis arthritis. Clin Rheumatol 2015; 34: 61-69, DOI: 10.1007/s10067-014-2744-6.

8. Constantin T, Foeldvari I, Vojinovic J, et al. Two-year efficacy and safety of etanercept in pediatric patients with extended oligoarthritis, enthesitis-related arthritis, or psoriatic arthritis J Rheumatol 2016; 43: 816-824, DOI: 10.3899/jrheum.150430.

9. Becker I, Horneff G. Risk of serious infection in juvenile idiopathic arthritis patients associated with tumor necrosis factor inhibitors and disease activity in the German Biologics in
Pediatric Rheumatology Registry. Arthritis Care Res (Hoboken) 2017; 69: 552-560, DOI: 10.1002/acr.22961.

10. Klotsche J, Niewerth M, Haas JP, et al. Long-term safety of etanercept and adalimumab compared to methotrexate in patients with juvenile idiopathic arthritis (JIA). Ann Rheum Dis 2016; 75: 855-861, DOI: 10.1136/annrheumdis-annrheumdis-2014-206747.

11. Cabrera N, Lega JC, Kassai B, et al. Safety of biological agents in paediatric rheumatic diseases: a real-life multicenter retrospective study using the JIR-cohorte database. Joint Bone Spine 2019; 86: 343-350, DOI: 10.1016/j.jbspin.2018.08.003.

12. Petty RE, Southwood TR, Manners P, et al. International League of Associations for Rheumatology classification of juvenile idiopathic arthritis: second revision, Edmonton, 2001. J Rheumatol 2004; 31: 390-392.

13. Crépin S, Villeneuve C, Merle L. Quality of serious adverse events reporting to academic sponsors of clinical trials: far from optimal. Pharmacoepidemiol Drug Saf 2016; 25: 719-724, DOI: 10.1002/pds.3982.

14. Samant SA, Campbell RL, Li JT. Anaphylaxis: diagnostic criteria and epidemiology. Allergy Asthma Proc 2013; 34: 115-119, DOI: 10.2500/aap.2013.34.3630.

15. Tarkiainen M, Tynjälä P, Vähäsalo P, Lahdenne P. Occurrence of adverse events in patients with JIA receiving biologic agents: long-term follow-up in a real-life setting. Rheumatology (Oxford) 2015; 54: 1170-1176, DOI: 10.1093/rheumatology/ keu457.

16. Common Terminology Criteria for Adverse Events (CTCAE) Version 4.0. http://evs.nci.nih.gov/ftp1/CTCAE/CTCAE_4.03_2010-06-14 Quick Reference_8.5x11.pdf

17. Lichtenstein L, Ron Y, Kivity S, et al. Infliximab-related infusion reactions: systematic review. J Crohns Colitis 2015; 9: 806-815, DOI: 10.1093/ecco-jcc/jjv096.

18. Chitnavis M, Stein DJ, Commins S, et al. First-dose anaphylaxis to infliximab: a case of mammalian meat allergy. J Allergy Clin Immunol Pract 2017; 5: 1425-1426, DOI: 10.1016/j.jaip. 2017.04.044.

19. Cansever M, Şahin N, Dursun I, et al. Successful slow desensitization to tocilizumab in a 15 -year-old patient. J Investig Allergol Clin Immunol 2018; 28: 436-438, DOI: 10.18176/jiaci.0314.

20. Galvão VR, Castells MC. Hypersensitivity to biological agentsupdated diagnosis, management, and treatment. J Allergy Clin Immunol Pract 2015; 3: 175-185, DOI: 10.1016/j.jaip.2014. 12.006.

21. Salmon JH, Perotin JM, Morel J, et al. Serious infusion-related reaction after rituximab, abatacept and tocilizumab in rheumatoid arthritis: prospective registry data. Rheumatology (Oxford) 2018; 57: 134-139, DOI: 10.1093/rheumatology/kex403.

22. Castells MC, Tennant NM, Sloane DE, et al. Hypersensitivity reactions to chemotherapy: outcomes and safety of rapid desensitization in 413 cases. J Allergy Clin Immunol 2008; 122: 574-580, DOI: 10.1016/j.jaci.2008.02.044.

23. Soyer O, Demir S, Bilginer $\mathrm{Y}$, et al. Severe hypersensitivity reactions to biological drugs in children with rheumatic diseases. Pediatr Allergy Immunol 2019; 30: 833-840, DOI: 10.1111/ pai.13114. 
24. Behrens EM, Beukelman T, Paessler M, Cron RQ. Occult macrophage activation syndrome in patients with systemic juvenile idiopathic arthritis. J Rheumatol 2007; 34: 1133-1138.

25. Barut K, Yücel G, Sinoplu AB, et al. Evaluation of macrophage activation syndrome associated with systemic juvenile idiopathic arthritis: single center experience over a one-year period. Turk Pediatri Ars 2015; 50: 206-210, DOI: 10.5152/TurkPediatriArs.2015.3299.
26. Tsuchida Y, Sumitomo S, Shoda H, et al. Macrophage activation syndrome associated with tocilizumab treatment in adult-onset Still's disease. Mod Rheumatol 2017; 27: 556-557, DOI: 10.1080/14397595.2016.1221875.

27. Handa R, Upadhyaya S, Kapoor S, et al. Tuberculosis and biologics in rheumatology: a special situation. Int J Rheum Dis 2017; 20: 1313-1325, DOI: 10.1111/1756-185X.13129. 